livraisons

d'Histoire

de l'Architecture

\section{Livraisons de l'histoire de l'architecture}

35 | 2018

Femmes, architecture et paysage

\title{
Deux cuisines médiatisées et leur transgression de genre : le cas de Bernège et Hefner
}

Two popularized kitchens and their transgression of kind: the case of Bernège and Hefner

Zwei prominente Küchenmodelle und ihre geschlechtsübergreifende

Konzeption : das Beispiel von Bernège und Hefner

Florencia Fernandez Cardoso

\section{(2) OpenEdition}

Journals

Édition électronique

URL : https://journals.openedition.org//ha/968

DOI : $10.4000 /$ lha. 968

ISSN : 1960-5994

Éditeur

Association Livraisons d'histoire de l'architecture - LHA

Édition imprimée

Date de publication : 15 juin 2018

Pagination : 99-109

ISSN : 1627-4970

Référence électronique

Florencia Fernandez Cardoso, « Deux cuisines médiatisées et leur transgression de genre : le cas de Bernège et Hefner », Livraisons de l'histoire de l'architecture [En ligne], 35 | 2018, mis en ligne le 15 juin 2020, consulté le 12 octobre 2021. URL : http://journals.openedition.org//ha/968 ; DOI : https:// doi.org/10.4000//ha.968 


\section{DEUX CUISINES MÉDIATISÉES ET LEUR TRANSGRESSION DE GENRE : LE CAS DE BERNĖGE ET HEFNER}

\section{Une cuisine à soi}

Une distinction genrée de l'espace émerge au XIX ${ }^{\mathrm{e}}$ siècle dans les milieux bourgeois et traditionnels en Europe ${ }^{1}$. Les rôles assignés selon le genre des individus étaient liés à l'espace public et privé : l'extérieur, ou l'espace public, était un monopole de la masculinité tandis que l'intérieur, ou l'espace domestique, était réservé aux femmes. D'après ce modèle connu comme les sphères séparées, l'espace public était désigné au travail et à la politique, domaines propres à la masculinité. L'intérieur privé, était un lieu "naturellement féminin ", la sphère où les femmes s'occupaient des enfants, de la cuisine et du ménage. Ces sphères séparées se retrouvaient aussi dans les revues de l'époque. Les revues "masculines " étaient associées aux sports, au travail, aux sciences et à la politique ; les revues "féminines " à la mode, à l'éducation des enfants et à la domesticité. En 1929, la célèbre écrivaine anglaise, Virginia Woolf, remarquait cette séparation genrée de l'espace dans son essai féministe intitulé "Une chambre à soi " ${ }^{2}$. Elle réclamait ainsi que, pour être intellectuellement libre, la «femme " puisse s'isoler dans un espace à soi lui permettant de réfléchir ou d'écrire.

Partant de l'idée d'un "espace à soi ", cet article propose de considérer la cuisine comme un espace d'épanouissement pour les femmes et les hommes, et ce, à travers les conceptions de deux personnalités, Paulette Bernège, la scientifique du ménage, et Hugh Hefner, le pornographe, Ni l'un ni l'autre n'étaient architectes, mais tous les deux ont influencé l'opinion publique en diffusant de nouvelles formes d'habiter. Les propositions publiées dans les revues qu'ils ont dirigées, respectivement Mon chez moi et Playboy, dérogeaient au modèle des sphères séparées à travers de nouvelles formes chorégraphiées de l'espace à soi. Bernège et Hefner étaient fascinés par la modernité et par l'architecture domestique. Ils se sont succédés à deux décennies d'écart et vivaient dans deux pays différents : la France et les États-Unis. Cependant, ils ont directement contribué aux nouveaux idéaux domestiques des années 1950 et au basculement des rôles de genre des années 1960, à travers leurs écrits et leurs imaginaires.

1. Laure Bereni et al., Introduction aux études sur le genre, $2^{\mathrm{e}}$ édition revue et augmentée, Brussels, De Boeck, 2012, p. 220-21.

2. Virginia Woolf, A Room of One's Own, Ebook, 2015 University of Adelaide, 1929. 


\section{Bernège et la cuisine de l'architecte-ménagère}

Paulette Bernège, née en 1896 dans la région d'Agen, était philosophe de formation. En 1923, elle est devenue rédactrice en chef de "Mon chez moi", une revue publiée de 1923 à 1930 qui avait près de vingt mille abonnés. Elle a aussi édité la revue du Salon des Arts Ménagers, L'Art Ménager ${ }^{3}$. En Belgique, elle a régulièrement écrit pour La Maison et pour le quotidien national le XXe Siècle. En 1924, elle a fondé et présidé la Ligue d'organisation Ménagère ${ }^{4}$. Bernège a publié de nombreux livres qui ont encouragé les architectes et les femmes à repenser l'espace domestique en France et en Belgique; les plus influents ont été De la Méthode Ménagère et Si les femmes faisaient les maisons ${ }^{5}$. Bernège a ainsi initié ses lecteurs français et belges au taylorisme ménager par ses publications, mais aussi par la fondation de plusieurs écoles spécialisées en science domestique. Elle a étudié l'aménagement d'une cuisine rationnelle à travers les mouvements et les activités culinaires. $\grave{A}$ partir de ses propres recherches et en s'inspirant du travail de Christine Frederick et Frederick Taylor, elle a appliqué les théories de l'économie du travail à l'espace ménager. Elle considérait que l'entretien domestique était un véritable travail, ce qui exigeait de lui trouver une formule d'organisation spécifique. Pour ce faire, elle a énuméré, transcrit et analysé l'effort et le temps que prenaient différentes tâches ménagères (cuisine, lavage, blanchissage, nettoyage, etc.). Par la suite, elle a comparé leur récurrence et leur emplacement dans l'ensemble du foyer et publié ses résultats dans Si les femmes faisaient les maisons:

"Les calculs d'heures perdues qui vont suivre, feront sûrement sourire les hommes superficiels et les femmes oisives, mais les statistiques officielles nous apprennent que plus de 5 millions de femmes françaises gagnent leur vie en dehors de leur maison et que, pour elles, le travail ménager vient en complément d'une journée bien remplie. Les hommes d'État, Monsieur Loucheur, n'ont pas le droit de sourire ${ }^{6}$."

Ses calculs lui ont permis de penser la cuisine de manière scientifique et de rationnaliser cet espace pour délimiter le temps perdu. Elle a théorisé l'aménagement d'une cuisine rationnelle en étudiant les mouvements et les activités culinaires. Comme une urbaniste qui étudierait la mobilité d'une ville et les distances entre les pôles urbains, elle proposait quatre pôles d'activités : préparation, cuisine, service et lavage, ainsi que deux espaces avoisinants et complémentaires des activités de la cuisine, la salle à manger et l'escalier de service. Le mur opposé au fourneau

3. Mary McLeod, Charlotte Perriand, An Art of Living, New York, Harry N. Abrams, 2003, p. 271.

4. Jackie Clarke, "L'organisation ménagère comme pédagogie : Paulette Bernège et la formation d'une nouvelle classe moyenne dans les années 1930 et 1940 ", Travail, genre et sociétés, 13, n 1, 2005, p. 139-57, https://doi.org/10.3917/tgs.013.0139.

5. Paulette Bernège, De la méthode ménagère, $2^{\mathrm{e}}$ éd. Paris, Mon chez moi, 1934 (1928); Paulette Bernège, Si les femmes faisaient les maisons, Paris, Mon chez moi, 1928.

6. Bernège, Si les femmes faisaient les maisons, p. 13. 


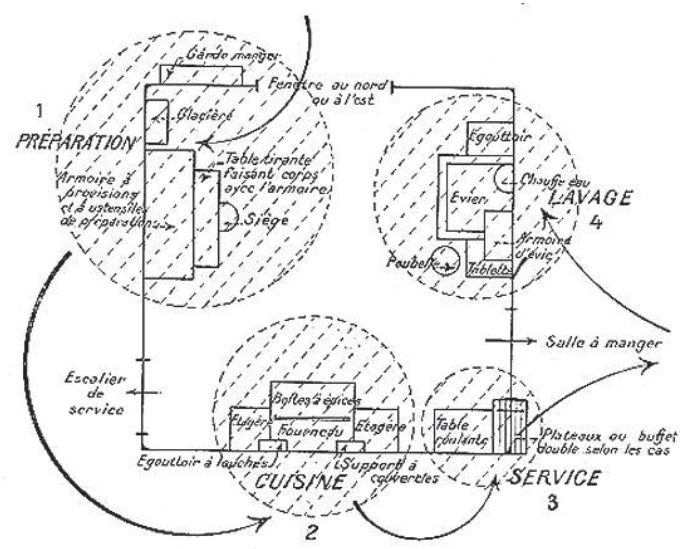

Ill. 1 : Plan de la cuisine rationnelle selon Bernège. Paulette Bernège, "L'Installation de La Cuisine ", La Construction Moderne, 44, no 22, 2 juin 1929, p. 437. (C) D.R.

devait avoir une fenêtre orientée au nord ou à l'est pour avoir une lumière modérée. Tous les éléments de la cuisine avaient une place spécifique. Elle en publia un schéma à deux reprises, dans le numéro de juin 1929 de la revue La Construction Moderne et, neuf ans plus tard, dans son Encyclopédie de la vie familiale (ill. 1).

Dès son premier livre De la méthode ménagère, Bernège définissait deux pièces de travail ménager par excellence : la cuisine et la salle de bains ${ }^{8}$. Dans cette publication figurent des dessins d'architecture de son ancien appartement parisien et une étonnante illustration de sa propre cuisine (ill. 2). Cette cuisine de son appartement parisien demeure l'exemple type de l'espace rationnel et de l'application de ses théories. Ainsi, elle définissait des pôles d'activités principales et elle réduisait au minimum la distance entre la table de travail, le fourneau et l'évier ; ces trois éléments accompagnant trois pôles d'activité, la préparation, la cuisine et le lavage.

Bernège décrivait l'espace de la cuisine comme un espace réservé exclusivement à la préparation de repas et éventuellement à un repas en solitaire. La cuisine personnelle de Bernège était un exemple français de la cuisine destiné à inspirer les femmes ménagères, mais aussi à valoriser leur travail et simplifier leurs tâches journalières. Dans la deuxième édition revue et corrigée de son ouvrage, Bernège a ajouté deux autres modèles à suivre : "La Cuisine Moderne" sous l'appellation "Équipement intérieur d'une habitation " de Le Corbusier et Charlotte Perriand, exposée en 1929 au Salon des Arts Ménagers en Automne, et celle conçue par la ménagère américaine Christine Frederick en 1930.

La cuisine de Frederick semble avoir fasciné Bernège. En 1930, elle a fait un séjour de trois mois aux États-Unis où elle a rendu visite à sa collègue en gestion

7. Paulette Bernège, "l'Installation moderne des cuisines ", La Construction Moderne, vol. 44, $\mathrm{n}^{\circ}$ 31, 5 mai 1929, p. 389-392; Paulette Bernege, Encyclopédie de la vie familiale, 1938.

8. Bernège, De la méthode ménagère, p. 146. 


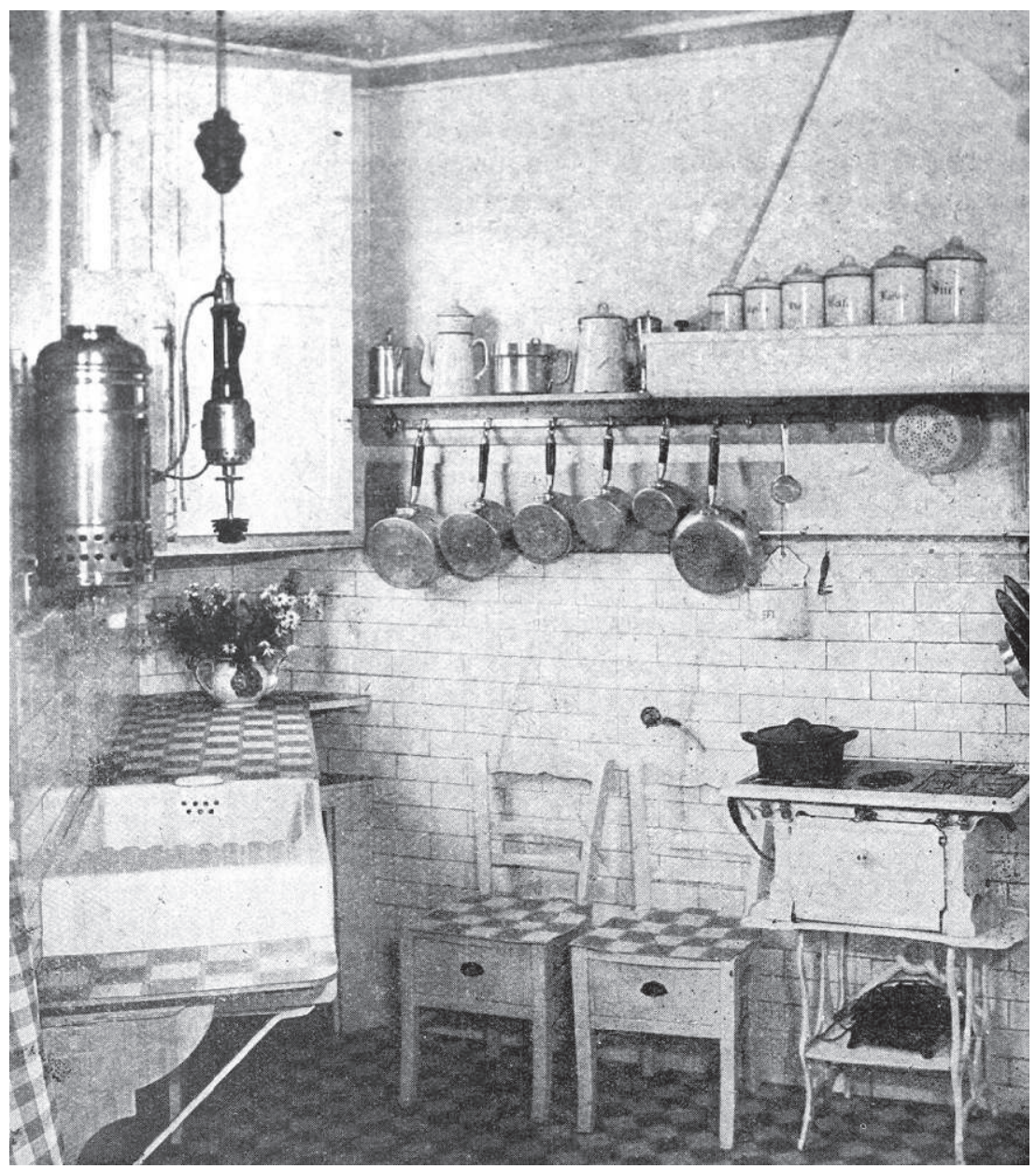

Ill. 2 : Photographie de la cuisine personnelle de Bernège dans son appartement 41, Boulevard RichardLenoir, 75011 Paris dans P. Bernège, De La Méthode Ménagère, 2 $2^{\mathrm{e}}$ éd. revue et corrigée, Paris, 1934, p. 140. (C) D.R.

ménagère. Frederick avait réussi à assimiler l'efficacité américaine au style architectural français. Après son retour en France, Bernège a publié cette cuisine dans plusieurs revues d'architecture : en France, La Construction Moderne, l'Art Ménager et l'Architecture d'Aujourd'hui; en Suisse, Habitation. En 1933, elle signa dans L'Art ménager un article qui cristallisait ses convictions ; "Quand une femme construit sa cuisine " ${ }^{9}$ reflétant sa valorisation du rôle des femmes dans la création de l'architecture et son appréciation de la cuisine comme un espace d'épanouissement personnel. Elle présentait sa collègue de l'habitat rationnel, Christine Frederick, comme une

9. Paulette Bernège, "Quand une femme construit sa cuisine ", 1933. 
"pionnière internationale ", laissant "apercevoir le nouvel essor américain qu'elle précède et qu'elle oriente". Son admiration pour la réalisation de Frederick peut être perçue comme un geste féministe valorisant la place des femmes dans la création architecturale.

Dès 1925, Bernège proposait d'élargir les rôles attribués aux femmes, francophones et de classe moyenne, pour leur inspirer l'idée de devenir des architectes ménagères. Même si elle n’incitait pas directement ses lectrices à lutter contre l'image de genre féminin de l'espace domestique, elle leur ouvrait la voie vers de nouvelles sphères. Elle leur suggérait de s'orienter vers la profession d'architecte ménagère, une nouvelle profession qui présentait «à la fois l'attrait de la création, la certitude d'une utilité sociale s'étendant sur plusieurs générations et l'espoir de revenus confortables. ${ }^{10}$ " Elle en justifiait l'importance : "Les architectes d'ordinaire se contentent de construire les maisons, écrivait-elle. C'est à peine si quelques-uns très modernes se soucient du travail que la femme aura à accomplir dans son intérieur... Il y a là tout un monde nouveau à étudier qui présentera pour la jeune fille intelligente, à l'esprit inventif, l'attrait des créations, en même temps que la certitude d'être pratiquement utile à toutes les femmes qui peinent dans leur foyer faute d'une bonne installation de leurs maisons ${ }^{11}$. " Elle a poursuivi cette valorisation de la femme dans la création architecturale non seulement dans sa publication Si les femmes faisaient les maisons, mais aussi dans le numéro de 1925 de Mon chez moi:

«Plus de paroles, plus de lamentations, plus d'indifférence. Au travail ! Plus que jamais les hommes ont besoin de la pondération, du sens pratique, du travail productif, patient et ordonné des femmes. À la désorganisation qui nous entoure, opposons la barrière inébranlable de l'organisation de nos foyers et du travail féminin ${ }^{12}$."

De cette manière, Paulette Bernège transgresse le modèle des sphères séparées en présentant l'espace domestique et la cuisine comme un lieu de travail et en incitant les femmes de foyer (son lectorat) à rejoindre la sphère publique pour pratiquer en tant qu'architectes-ménagères.

\section{Hefner et la cuisine pour célibataires}

Deux décennies plus tard, un américain a suivi ses conseils et incité les hommes à s'investir à leur tour dans la conception du logement. Après la deuxième guerre mondiale, en 1949, Hugh Hefner a obtenu sa licence en psychologie et fondé une famille avec une amie de l'université d'Illinois. Il était né à Chicago en 1926 et

10. Marie-Jeanne Dumont, "Si les femmes faisaient les maisons ", La Croisade de Paulette Bernège, Criticat, ${ }^{\circ} 10$, Fall, 2012, p. 60.

11. Paulette Bernège, "Les Architectes Ménagères ", Mon chez moi, Modern Living, XXI, mai 1925, p. 61.

12. Bernège, ibid., "Les Architectes Ménagères ". 
c'est aussi là qu'il a commencé sa carrière dans Lifestyle et, en tant que dessinateur, dans la revue masculine Esquire. En 1953, il a fait l'objet d'un article publié dans le Chicago News: "How a Cartoonist Lives ". ${ }^{13}$ À l'opposé de l'image qu'on peut avoir d'Hefner aujourd'hui, de propriétaire d'un empire pornographique, on perçoit un homme blanc traditionnel qui travaille pour soutenir sa femme et son nouveauné. Un an plus tard, voit le jour le magazine Playboy, la première étape de la constitution de son entreprise.

Quelques décennies après Bernège, Hefner a contesté, à son tour, la vision genrée du logement, en défendant le droit de l'homme à l'espace domestique et en proposant la cuisine comme un lieu de divertissement ${ }^{14}$. Avec la revue Playboy, il a développé une nouvelle conception de la domesticité moderne et de la masculinité américaine des années 1950 qui transgressait l'image traditionnelle de l'homme marié. Il s'intéressait à un nouvel espace quotidien, pas encore reconquis par les hommes de l'époque, celui de la cuisine moderne.

Dès les débuts de Playboy, Hefner a voulu la distancier des autres revues pour hommes de l'époque. Le premier numéro, publié en 1953, indique que Playboy n'est pas une revue comme les autres parce qu'elle propose au lecteur de passer le plus clair de son temps chez lui, à l'intérieur de son appartement d'homme moderne. De 1953 jusqu'aux années 70, Hefner y a présenté une large variété de projets domestiques ou d'appartements pour hommes célibataires, la cuisine pour célibataires étant un thème central. En quelques années, la revue a ainsi dévoilé des architectures domestiques comme l'Appartement Playboy Penthouse ${ }^{15}$, le lit Playboy ${ }^{16}$ et la Kitchenless-Kitchen ou la cuisine-non-cuisine du Playboy ${ }^{17}$ (ill. 3).

L'espace domestique proposé par Hefner n'était pas rationnel, comme celui de Bernège, mais il était conçu pour faciliter une rencontre sexuelle. Le premier projet qui le démontra est le "Progrès du Playboy", publié en mai $1954^{18}$. Ce projet était présenté par un schéma instructif garantissant une conquête en 25 étapes. L'objectif était de guider l'invitée, une femme affamée, dans le lit de l'amphitryon. Comme l'avait fait Bernège, l'auteur de l'article décrivait les mouvements et les tâches à suivre pour arriver au but de la manière la plus efficace. Au lieu de rechercher une économie domestique, Hefner composait un décor et une scénographie réfléchie et détaillée pour faciliter la séduction de façon inaperçue. Il n'appliquait pas des théories scientifiques ménagères, mais s'inspirait d'Alfred Kinsey qui avait publié un an plus tôt un rapport sur le comportement sexuel masculin ${ }^{19}$.

13. William Kiedaisch, "How a Cartoonist Lives », Chicago Daily News, 21 March 1953, p. 8.

14. Beatriz Colomina, Ann-Marie Brennan, and Jeannie Kim, eds., Cold War Hothouses : Inventing Postwar Culture, from Cockpit to Playboy, New York, Princeton Architectural Press, 2004.

15. "Playboy's Penthouse Apartment ", Playboy, Modern Living, 3, no. 9, September 1956, p. 53-60 ; "Playboy's Penthouse Apartment ", Playboy, Modern Living, 3, n. 10, October 1956, p. 65-70.

16. "The Playboy Bed ", Playboy, Modern Living, 6, no. 11, November 1959, p. 66-67 et p.107.

17. "The Kitchenless Kitchen ", Playboy, Modern Living, 6, nº 10, October 1959, p. 53-55 et p. 108.

18. Hugh Hefner, ed., "Playboy’s Progress ", Playboy 1, nº. 6, May 1954, p. 23-24.

19. Alfred Charles Kinsey et al., Sexual Behavior in the Human Female, Institute for Sex Research, Indiana University Press, 1953. 


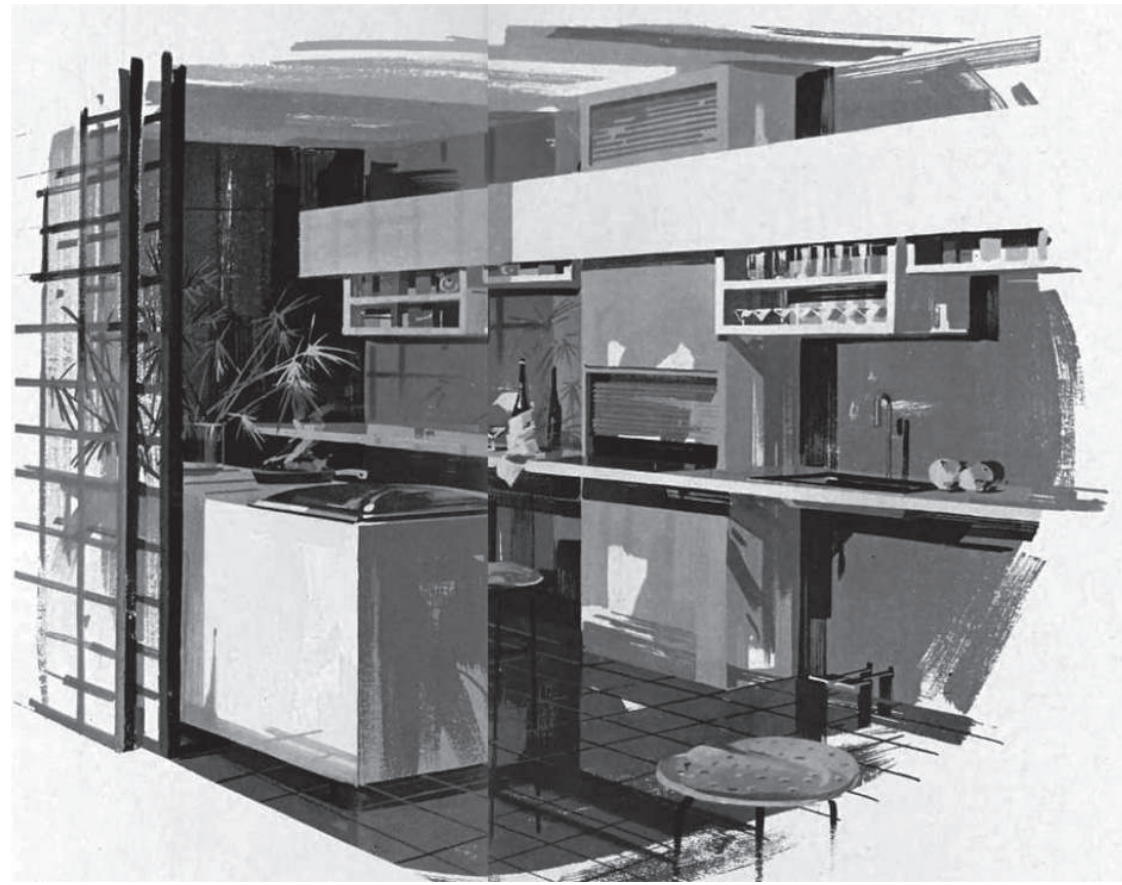

Ill. 3 : Illustration de la cuisine dans l'article de Playboy, "Playboy's Penthouse Apartment ", Playboy, Modern Living, 3, no 9, septembre 1956, p. 58-59. (C) D.R.

Dans cette chorégraphie de l'habitat, la cuisine jouait un rôle essentiel. Tout d'abord, l'invitée entrait dans le logement avec l'espérance d'un repas après le théâtre. Le playboy avait déjà préparé des ailes de poulet à déguster avec les doigts. Dans les vingt-cinq étapes que comportait le processus, la femme affamée et le playboy se retrouvaient par trois fois dans la cuisine (points 5, 13 et 17). Le playboy ayant préparé le dîner, mais ne proposant pas de manger à table, les deux acteurs étaient constamment obligés de revenir à la cuisine. De plus, il préparait constamment des cocktails, ce qui facilitait la conquête, jusqu'au moment où l'invitée, qui hésitait une dernière fois à retourner à la cuisine pour chercher à manger (point 24), décidait plutôt de suivre le playboy jusqu'à son lit.

Le manuel de séduction au sein de l'espace domestique et de la cuisine a été poursuivi durant la première décennie de la revue, avec les projets d'habitat pour homme célibataire. En mai 1956, Playboy a publié le penthouse du playboy, parce que "l'homme aspire à un habitat à soi ". L'homme rêverait ainsi d'avoir son propre domaine domestique, un espace qui serait "plus qu'un endroit pour déposer son chapeau, un espace exclusif à lui. ${ }^{20}$ "Comme si elle reprenait les mots de Virginia Woolf, la revue laissait entendre que l'homme de Playboy avait besoin d'un espace 
à lui pour échapper à la société américaine de l'époque qui se focalisait sur le noyau familial. À travers le design d'espace domestique, Hefner transgressait les rôles de genre de l'époque.

La cuisine était séparée de la salle à manger par six panneaux coulissants, comme des panneaux translucides japonais, qui permettaient de fermer ou d'ouvrir complètement la cuisine. L'installation de la cuisine était pensée " pour être efficace et pour réduire au maximum les complications et le travail ménager ${ }^{21}$. » Le travail ménager était décrit comme le "Hausfrau labor ", le travail de femme au foyer, le playboy méprisant les activités qui viennent après la préparation du repas, la cuisson et la présentation. Les activités de rangement et nettoyage qui prenaient plus de temps, n'étaient pas censé être intéressantes pour le célibataire:
"Parce que ceci est une cuisine pour l'homme célibataire, rappelez-vous, et à moins que vous soyez un célibataire très excentrique, vous aimez cuisiner et préparer promptement des spécialités rapides au même degré que vous aimez faire la vaisselle, ranger et nettoyer. Tout ça a été réglé ici. ${ }^{22}$ "

Ainsi, dans la cuisine du penthouse, tous les équipements et installations de dernière technologie étaient intégrés pour que l'homme célibataire ne perde pas son temps après le repas. La quatrième activité de Bernège, le lavage, était supprimée et confiée aux machines : un lave-vaisselle, qui était aussi un élément mobilier, imaginé pour offrir une simplicité du travail de nettoyage. Ainsi, le playboy remplaçait la femme au foyer par toutes les technologies possibles et encore peu répandues afin de transgresser la limite genrée, et il s'appropriait la sphère domestique. L'espace de cuisine devenait un espace de divertissement pour le célibataire.

Le projet de cuisine pour célibataire a été encore enrichi, plus de trois années plus tard, par la "cuisine-non-cuisine", publiée en 1959. Celui-ci proposait un développement de la cuisine imaginée pour l'appartement penthouse du playboy. La «cuisine-non-cuisine" était plus compacte et plus réaliste que la précédente. Elle était réduite à un îlot central, "une péninsule " faite d'une table de travail pour la préparation, la cuisson et le service du repas. À partir d'un couvercle pivotant, cet îlot pouvait se fermer et s'étendre pour accueillir des invités, devenant une table-bar (ill. 4).

La péninsule de cette "cuisine-non-cuisine " avait une forme rectangulaire de 2,10 m par 0,60 m, et était adossée à un mur. Dans un même meuble, on retrouvait le réfrigérateur, l'évier et toute sortes des machines électriques pour garantir la cuisson du repas. Le projet était décrit en ces termes :

"Quelles que soient les circonstances, la cuisine-non-cuisine transforme le grignotage ou le festin en un jeu d'enfant et un régal. Ce beau morceau de

21. Ibid., p. 60 .

22. Ibid., p. 60. "For this is a bachelor kitchen, remember, and unless you're a very odd-ball bachelor indeed, you like to cook and whomp up short-order specialties to exactly the same degree that you actively dislike dishwashing, marketing and tidying up. All that's been taken care of here. » 


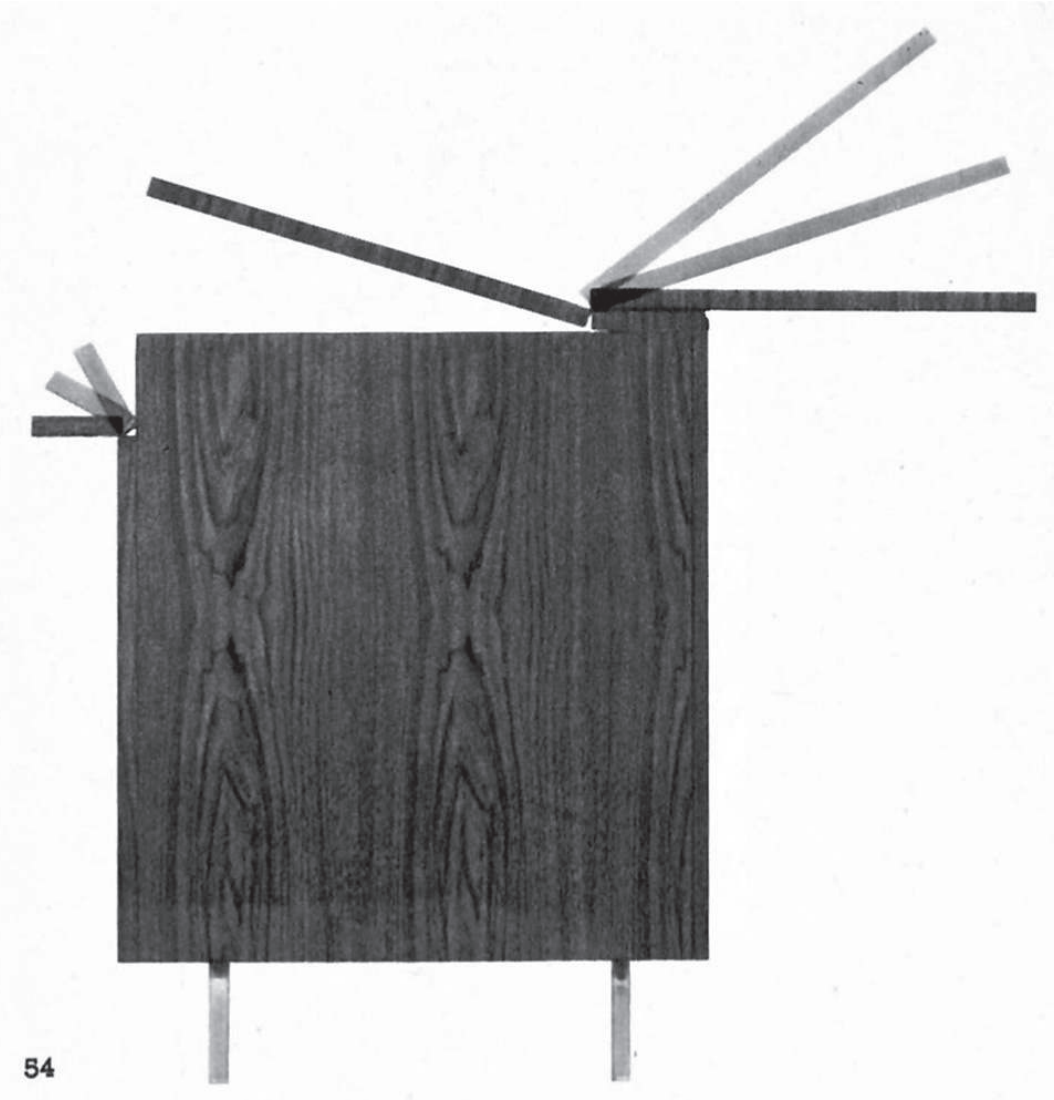

Ill. 4: Photographie avec les mouvements possibles de la cuisine-non-cuisine, "The Kitchenless Kitchen ", Playboy, Modern Living, 6, n 10, octobre 1959, p. 54. (C) D.R.

meuble, conçu par PLAYBOY, propose une cuisine en entier et rend le proverbial fourneau inutile; avec ce mobilier, on n'a plus besoin de la collection habituelle des casseroles, poêles, poêlons et autres équipements de cuisine ${ }^{23}$."

Cette cuisine était présentée avec un scénario similaire à celui qui accompagnait la publication de l'appartement penthouse du playboy : après le théâtre, des femmes affamées viendraient chez le célibataire pour " un banquet à minuit ", quelque chose de "plus détendu» que les "foules des restaurants ». La "cuisine-non-cuisine »

23. "The Kitchenless Kitchen ", p. 54. "Whatever the circumstances, the kitchenless kitchen makes snacking or feasting a cinch and a treat. This handsome hunk of furniture, designed by PLAYBOY, dispenses with a kitchen as such entirely; it renders the proverbial hot stove unnecessary; it has no use for the usual collection of pots, pans, skillets, over and other customary kitchen gear." 
devenait le centre d'attention de l'appartement, elle fonctionnait comme un podium sur lequel le playboy pouvait jouer le rôle de chef de cuisine et séduire de nouvelles invitées. Dans les séries d'articles catégorisés "Food and Drink " et "Modern Living ", la revue proposait régulièrement des recettes de cuisine et des projets d'architecture domestique pour inviter l'homme à exploiter et aménager son propre espace de cuisine. Dans ces projets dessinés pour les "hommes célibataires ", la consommation et la domesticité n'étaient plus exclusivement réservées aux ménagères. En 1959, trois décennies après Bernège, Playboy avait réussi à former une identité masculine autour de la cuisine du célibataire, ancrée dans l'architecture moderne et l'espace public des villes modernes américaines comme Chicago et New York.

\section{Les cuisines médiatisées qui épanouissent}

Entre 1925 et 1940, Paulette Bernège a contesté, dans ses articles et dans ses ouvrages, la vision genrée et réductrice de la domesticité. Elle a incité les architectes à considérer la valeur des femmes architectes et les femmes ménagères à s'investir dans la création de l'architecture. Quelques décennies plus tard, Hugh Hefner a lui aussi contesté la vision genrée de la domesticité dans sa revue Playboy, en défendant le droit de l'homme à l'espace domestique. Avec leurs conceptions, ils ont incité à la transgression des sphères attribuées aux genres.

En interprétant les tâches ménagères comme un travail digne d’étude et d'efficacité, Bernège a, d'une certaine manière, rehaussé le statut des "femmes ménagères " de classe moyenne. Sa démarche visait à l'émancipation de la simple ménagère, en la considérant comme une professionnelle respectable, une " directrice de la gestion du ménage ». Elle montrait la valeur économique du travail ménager, estimant que « la France oblige ses ménagères à perdre par année 7 milliards 300 millions d'heures ${ }^{24}$ ". C'était, plus que du temps perdu pour la femme, de l'argent perdu pour toute l'économie française. À l'opposé de Bernège qui insistait pour que les femmes deviennent des " architectes-ménagères ", le créateur de l'empire Playboy associait l'identité masculine à l'architecture moderne et à la profession de l'architecte. En utilisant Playboy comme un medium au service des architectes modernes masculins, par le biais d'interviews et de présentations de leurs projets, Hefner s'est servi de l'architecture moderne pour développer son idéologie de l'identité masculine divertissante. Sa vision de la cuisine et de l'architecture domestique est définie complétement à l'écart de la femme. Sa revue laissait penser que l'architecture moderne et l'espace de la cuisine étaient une affaire réservée exclusivement aux hommes.

En 1928, les participants du premier des congrès internationaux d'architecture moderne (CIAM), déclaraient qu'il était « indispensable que les architectes exercent une influence sur l'opinion publique en lui faisant connaître les bases de l'architec-

24. Bernège, Si les femmes faisaient les maisons, p. 5. 
ture nouvelle ${ }^{25}$. " Paulette Bernège et Hugh Hefner n'étaient pas des architectes, mais ils ont tous deux répondu à ce défi. Ils ont formé des théories et des étapes à suivre dans la vie domestique selon leurs idéologies genrées. Avec leurs conceptions de l'espace domestique et de la cuisine moderne, ils ont inspiré une transgression des rôles assignés à chaque sexe.

Florencia FERNANDEZ CARDOSO Architecte et doctorante en cotutelle ULB-KU Leuven

25. Congrès Internationaux d'Architecture Moderne (C.I.A.M.), "Déclaration de La Sarraz ", Habitation 41, no 2, 1928-1968, p. 16, https://doi.org/10.5169/seals-126406. 
